Abstract 28 Table 1 Edwards Sapien, Abbott Portico, Medtronic Evolut and NVT Allegra valve size choices using heart navigator III vs CT human-operator

\begin{tabular}{|l|c|c|c|c|c|}
\hline & \multicolumn{5}{|c|}{ Valve Size Chosen (\%) } \\
\hline & -1 & Same & +1 & +2 & +3 \\
\hline Edwards Sapien & 16.3 & 71.3 & 11.9 & 0.6 & - \\
\hline Abbott Portico & 20.0 & 60.6 & 18.8 & - & 0.6 \\
\hline Medtronic Evolut & 15.6 & 71.3 & 12.5 & 2.3 & 0.6 \\
\hline NVT Allegra & 16.9 & 68.8 & 13.8 & 0.6 & - \\
\hline
\end{tabular}

Background Transcatheter aortic valve implantation (TAVI) requires assessment of gated CT images for accurate aortic annulus sizing. We investigated the accuracy of The Heart Navigator III software (Philips Healthcare, Netherlands) in performing fully automatic annulus measurements.

Methods One-hundred and sixty patients underwent gated cardiac CT scans as pre-assessment for a TAVI procedure. The Heart Navigator III software (Philips Healthcare, Netherlands) performed automatic segmentation of the aortic root and measurement of the aortic annulus area in systole without operator intervention. These were compared with manual measurements made by an experienced CT operator during pre-procedural planning with commercially available CT software. We then evaluated whether the automated measurements would lead to the same valve size selected as the human-operator utilising commonly used TAVI manufacturers.

Results When Heart Navigator III automatic measurements of the aortic annulus size were compared to CT human-operator images, there was a bias of $-1.48 \mathrm{~mm}^{2}$. 95\% limits of agreement were from -96.16 to $+93.21 \mathrm{~mm}^{2}$ (see figure 1). Automatic measurements and CT human-operator measurements led to the same size Edwards Sapien valve in $71.3 \%$ of patients, Abbott Portico valve in $60.6 \%$ of patients, Medtronic Evolut in $71.3 \%$ of patients and NVT Allegra in $68.8 \%$ of patients. The Heart Navigator III selected valves within 1 size of the human-operator choice in $97.1-99.4 \%$ of cases (table 1).

Conclusion The Heart Navigator III software (Philips Healthcare, Netherlands) is a promising technology allowing fully automated aortic annulus segmentation and sizing. However,

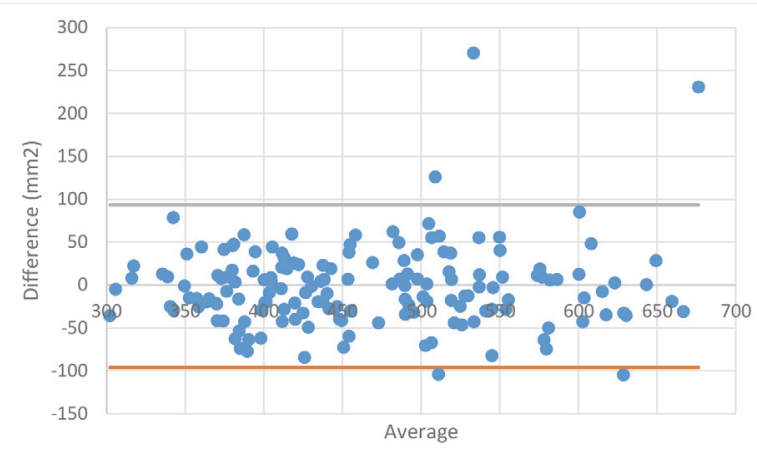

Abstract 28 Figure 1 Bland-Altman analysis demonstrating the difference between the heart navigator III measurements and CT operator measurements at present the accuracy is not sufficient for clinical use and human-operator oversight is still required.

Conflict of Interest None

\section{GONE BUT NOT FORGOTTEN: A CONTEMPORARY IMAGING SERIES OF PATIENTS WITH A SYSTEMIC RIGHT VENTRICLE AND A LV-PA CONDUIT FOR NATIVE OBSTRUCTION OF THE LEFT VENTRICULAR OUTFLOW TRACT.}

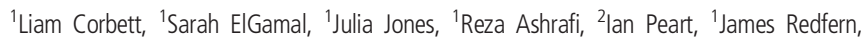
'Damien Cullington. 'Liverpool Heart \& Chest Hospital NHS Foundation Trust, Liverpool, UK; ${ }^{2}$ Alder Hey Children's Hospital NHS Foundation Trust

\subsection{6/heartjnl-2021-BCS.29}

Background In the UK, left ventricle to pulmonary artery (LVPA) conduit implantation was utilised in two centres in the late 1980 s to 1990 s in very small numbers. The LV-PA conduit bypassed native LV-PA outflow tract obstruction in patients with transposition of the great arteries (D-TGA) with an atrial-switch and congenitally corrected transposition of the great arteries (CCTGA, L-TGA). It is now generally appreciated that LV-PA obstruction offers some physiological advantage for patients with an atrial-switch or CCTGA to 'preserve' interventricular septal conformation and to help lessen progressive systemic tricuspid valve regurgitation - akin to 'physiological' repair with PA banding. The practice of LV-PA conduit implantation has essentially become extinct from clinical practice.

Case Presentations We report of 5 patients with native LV-PA obstruction that all underwent additional extra-cardiac LV-PA conduit implantation without resection of their native LV-PA obstruction. The operations were performed at the same surgical centre between 1989 and 1995 ( $n=3$ CCTGA, $n=2$ DTGA with atrial-switch). During adulthood follow-up, imaging of the LV-PA conduit was missed or deemed non-diagnostic by echocardiography. Subsequent cross-sectional imaging found all conduits to be small in calibre with an unusual anterior course. Patient $1 \& 2$ remained stable with preserved ventricular function, low sub-pulmonic left ventricular pressures and only mild gradients demonstrated through their LV-PA outflows. Patients 3, $4 \& 5$ had preserved ventricular function in the context of severe native and extra-cardiac LV-PA conduit obstruction, with significant circumferential conduit calcification and adhesion to the retro-sternum. Patient 3 underwent conduit excision and replacement after presenting with Staph Capitus endocarditis, which was confirmed with PET-CT, 
whilst Patient 4 underwent balloon dilatation and stenting, as the primary stenosis was discrete at the distal anastomosis site. After 6-months follow-up, Patient $3 \& 4$ recovered well with improved clinical status. Patient 5 had complete LV-PA conduit adhesion to the retro-sternum and demonstrated sub-systemic sub-pulmonary pressures by diagnostic catheterisation. It was agreed that the intervention risk outweighed any potential benefit and conservative management with follow-up would be most appropriate.

Discussion In our cases, despite knowing the exact anatomical location and conduit course, repeat echocardiography remained non-diagnostic. This surgical approach was first described in 1976, and there are case reports on only 15 patients, whereby, direct LV-PA resection was not felt to be surgically feasible due to abnormal mitral valve chordal attachments and/or long fibromuscular tunnel-type obstruction. It remains technically challenging to directly relieve native LV-PA obstruction without having detrimental impact on systemic right ventricular function, tricuspid valvular competence and electro-physiologically. But as ACHD practice has evolved, it is now actually an anatomical problem which doesn't necessarily always need a 'surgical-fix'.

Conclusion Awareness of this historical surgical approach to managing native LV-PA obstruction is important to understanding physiology and the potential long-term sequelae in these patients. They present a particular imaging challenge, especially via echocardiography, but an important finding to be comprehensively assessed through multimodal cross-sectional imaging. Although the technique is gone, it shouldn't be forgotten. Conflict of Interest None

\section{SEVERE AORTIC STENOSIS MANAGEMENT IN A TERTIARY CARDIAC CENTRE}

Norildin Al-Refaie, Waqas Jarral, Abhishek Shetye, Mark Cassar, Jim Newton. Oxford University Hospitals NHS Trust, Oxford, UK

\subsection{6/heartjnl-2021-BCS.30}

Introduction Aortic stenosis (AS) is the most common valvular heart disease in developed countries with an estimated prevalence of $3 \%$. According to ESC/EACTS guidelines, invasive management is recommended in patients with severe symptomatic AS, because of dismal spontaneous prognosis. It was reported that the average survival durations of patients with severe AS after developing symptoms such as angina, syncope, and shortness of breath were only 5, 3 and 2 years respectively if managed conservatively. Management of asymptomatic severe AS remains controversial.

Purpose Assess the prevalence of symptoms in patients with severe AS, and their management plans in relation with symptoms and age.

Method Retrospective data analysis of 259 patients with severe AS in a tertiary cardiac centre. Clinic notes, operation sheets, procedure reports and discharge summaries were the main source of data.

Results 199 (76.8\%) patients were symptomatic (shortness of breath, chest pain, dizziness or syncope). 143 (71.8\%) of these underwent intervention (surgical aortic valve replacement (SAVR), transcatheter valve implantation (TAVI) or Valvuloplasty). 56 (28.2\%) were managed conservatively (reasons for this being high risk for intervention, technical inadequacy for TAVI and patient informed decision). 9 (4.5\%) patients passed away during the study duration (all were managed conservatively).60 (23.2\%) patients were asymptomatic, of which 8 $(13.3 \%)$ underwent intervention. None of medically managed asymptomatic patients passed away during the study period. Average age for symptomatic patients who had surgical AVR

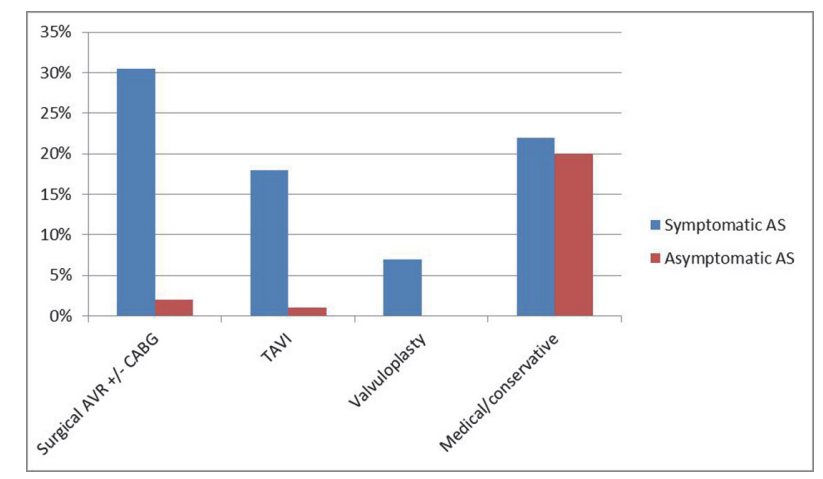

Abstract 30 Figure 1

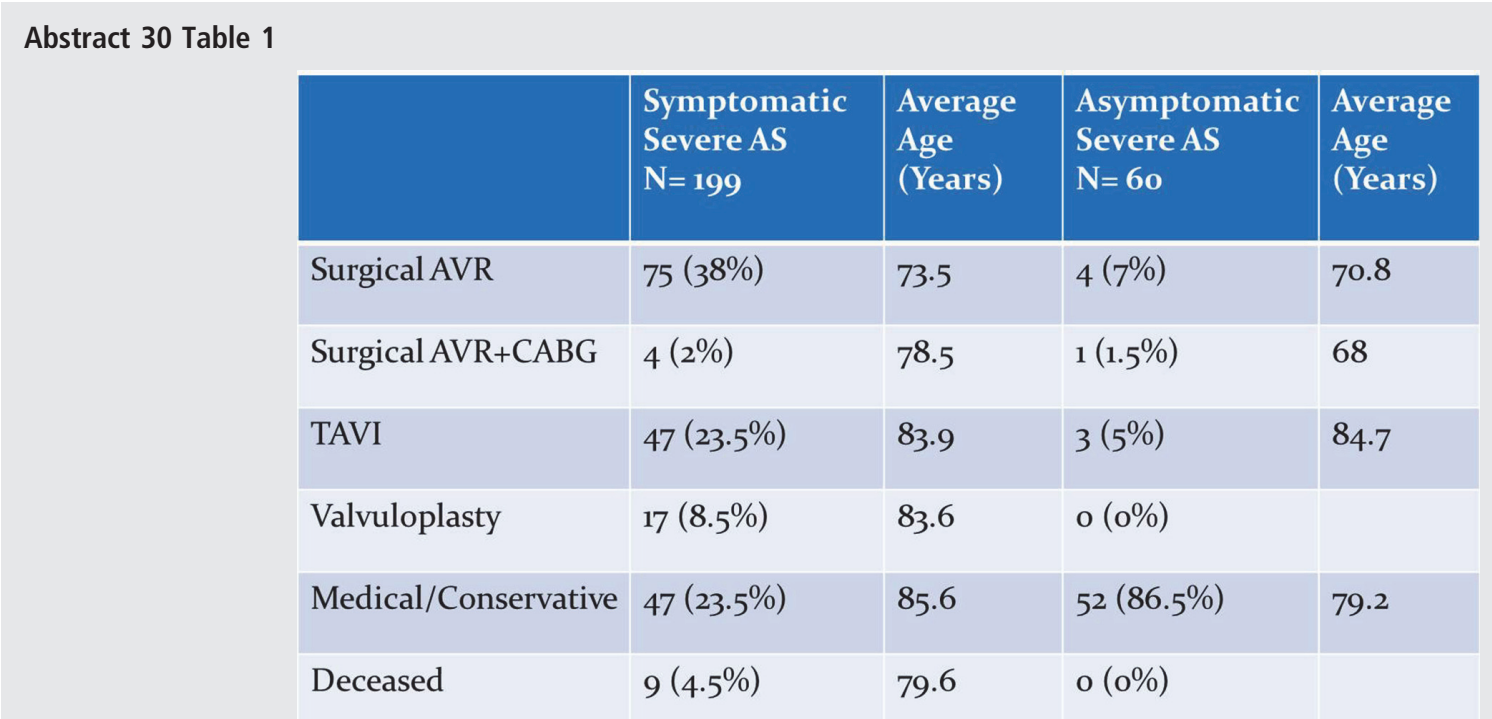

